\title{
Was kann Coaching von Mentoring lernen?
}

\author{
Anregungen aus der aktuellen Mentoring-Forschung
}

\author{
Friederike Höher
}

Online publiziert: 17. September 2015

(C) The Author(s) 2015. Dieser Artikel ist auf Springerlink.com mit Open Access verfügbar.

\begin{abstract}
Zusammenfassung Aus der Mentoring-Forschung und besonders einem aktuellen Ansatz zum vernetzten Lernen im Mentoring ergeben sich einige Anregungen für Coaching, seine Evaluation und Wirksamkeitsforschung: Während Evaluationen von Coaching seine Erfolge für die Coachees und zum Teil auch seine Auswirkungen auf das organisationale Umfeld berücksichtigen, thematisiert die neuere Mentoring-Forschung zunehmend ebenfalls die Auswirkungen des Mentorings auf die Mentor/innen und reflektiert damit eine Entwicklung des MentoringVerständnisses in Richtung wechselseitiger Lern- und Entwicklungsbeziehungen. Da die Mentorenrolle mit der des Coachs zum Teil vergleichbar ist, wird dieser Gedankengang hier für die Wirksamkeitsforschung von Coaching aufgegriffen.
\end{abstract}

Ausgangspunkt der Überlegungen ist eine Untersuchung zum vernetzten Lernen im Mentoring in einer Langzeitperspektive, durch die die Effekte des Mentorings für beide Seiten, Mentees und Mentorinnen sehr deutlich werden.

Schlüsselwörter Coaching · Mentoring - Evaluation · Wirksamkeitsforschung · Coaching-Evaluation · Mentoring-Evaluation · Wirksamkeit von Coaching

\section{What Coaching can learn from mentoring - conclusions from current research on mentoring}

Abstract There are a few interesting learnings from research on mentoring - specifically from a current approach

F. Höher $(\square)$

Dortmund, Deutschland

E-Mail: info@friederike-hoeher.de on networked learning in mentoring - that are valuable for a discussion on coaching, evaluation and its effectiveness in general: While the evaluation of coaching is taking into account its effects on coachees and partly on the organizational environment, the latest research on mentoring focuses on the repercussions for the mentors as well. It thereby reflects on the development of an understanding of mentoring that is taking mutual relationships for learning and development into account. Since the role of the mentor is similar to that of the coach in some aspects, the research on effectiveness of coaching is adapting this concept. The starting point for our considerations is a study on networked learnings in mentoring in a long term perspective - and the fact that it explicitly highlights the ramifications of mentoring for both sides, mentor and mentee.

Keywords coaching $\cdot$ mentoring $\cdot$ evaluation $\cdot$ research on effectiveness of mentoring and coaching

Coaching und Mentoring weisen neben Unterschieden auch viele Gemeinsamkeiten auf. Aus der Mentoring-Forschung und besonders einem aktuellen Ansatz zum vernetzten Lernen im Mentoring (Höher 2014) könnten sich einige Anregungen für die Coaching-Forschung ergeben.

\section{Zur Wirksamkeit von Coaching}

Im Gegensatz zum wachsenden Interesse der Wissenschaften an Coaching existiert nach wie vor ein Mangel an systematischen Studien zu seiner Wirksamkeit. Es gibt noch immer wenige, und erst recht wenige empirisch abgesicherte, Studien dazu, wie Coaching eigentlich wirkt (vgl. Greif 2009, 2012; Künzli 2006, 2009). 
Ein weitreichender und oft rezipierter Entwurf eines umfassenden Wirkmodells stammt von Greif (2008). Es beinhaltet neben der Prozessebene als weitere Bestandteile die Voraussetzungen des Coachs wie des Coachees sowie evaluierbare spezifische und allgemeine Ergebnisse des Coachings. Einige Evaluationen von Coaching beziehen den organisationalen Kontext (Personalentwicklung, Vorgesetzte) mit ein, in verschiedenen Untersuchungen wird Coaching aus dieser Sicht als erfolgreiche Maßnahme bestätigt (vgl. Künzli 2006). Weitere Arbeiten beschäftigen sich mit einer Nutzenquantifizierung von Coaching, indem sie den Return on Investment berechnen (vgl. Giacovelli und Goldkamp 2009). Die ermittelten Werte sind generell sehr hoch, sodass nachgewiesen zu sein scheint, dass sich eine Investition in Coaching für das beauftragende Unternehmen auszahlt. Coaching erzielt offensichtlich weltweit positive Wirkungen (Bresser 2012). Sie reichen - bei aller Vorsicht hinsichtlich Aussagekraft und Verallgemeinerbarkeit der Ergebnisse - von emotionaler Entlastung, Perspektivenwechsel und erhöhter Selbstreflexion bis zu verbesserter Führungskompetenz und Kommunikation sowie einem besseren Beziehungsverhalten und effektiverem Handeln (vgl. Künzli 2006, 2009; Greif 2008, 2012). Die CoachingForschung kann belegen, dass Coaching Persönlichkeitsentwicklung ermöglicht (Greif 2008) und nachhaltig wirkt (Künzli 2006).

Evaluationen von Coaching beinhalten ein grundsätzliches Dilemma: Abgesehen von einer Überschätzung der Aussagekraft von Zahlen zur Nutzenquantifizierung ist zu berücksichtigen, dass Coachees den Coaching-Prozess und seine Ergebnisse mitgestalten, sodass ihnen in der Konstruktion einfacher Kausalzusammenhänge eine Wirkungsillusion anhaften könnte. Wenn es auf die Frage wie Coaching wirkt aber noch keine verlässlichen Antworten gibt, ist mit Greif (2008) anzuregen, nicht nur in der ,schmalen Coachingforschung" (Greif 2008, S. 74) zu suchen, sondern auch in verwandten Forschungsfeldern nach vermutlich übertragbaren Erkenntnissen. U.a. Greif nutzt hierfür die Psychotherapieforschung. Ich rege an, sich darüber hinaus auch der reichhaltigen Mentoring-Forschung zuzuwenden und zu überprüfen, in wie weit sich hier vorgefundene Fragestellungen, Vorgehensweisen, Modelle und Ergebnisse für die Coaching-Forschung nutzbar machen lassen.

\section{Mentoring und Coaching}

Mentoring erfuhr in den USA bereits seit den 80er Jahren eine stets wachsende Bedeutung und ein entsprechendes Forschungsinteresse. Die Übergänge zu Coaching als Einzelcoaching durch die Führungskraft scheinen fließend und es ist daher schwierig, diese beiden Beratungsformate präzise voneinander abzugrenzen. In methodischer Hinsicht gleichen sich beide Verfahren, sie greifen auf ähnliche Interventionen und Grundüberzeugungen zurück (Brockband und McGill 2006; Garvey et al 2009), beide gelten als „learning relationships at the heart of change" (Connor und Pokora 2007, S. 7).

Im Kontext der Veränderung von Organisationen und den Lernanforderungen an ihre Mitglieder haben sich MentoringVerständnisse im Laufe der Jahrzehnte weiterentwickelt. Zunächst (und z. T. noch immer in der aktuellen CoachingLiteratur, vgl. z. B. Greif 2008) herrschte eine seinerzeit aus dem informellen Mentoring hervorgehende Auffassung von einer komplementären Beziehung vor, in der eine ältere, erfahrene Führungskraft eine jüngere orientiert, fördert, an implizitem Wissen (Erfahrungswissen) teilhaben lässt und in eine Organisation hinein sozialisiert. Später trugen Higgins und Kram (2001) zu einer Erweiterung des Mentoring-Verständnisses bei, indem sie eine „developmental network perspective“ einführten (Higgins und Kram 2001, S. 268), denn heute werden auch andere Perspektiven und Beziehungen als nur eine Senior-Perspektive für organisationales Lernen gebraucht. Damit rücken weitere und auch organisationsübergreifende Kontakte in den Blick: Nicht nur die exklusive Beziehung zu einem Mentor/einer Mentorin zählt für beruflichen Erfolg, Lernen und Problemlösen, sondern es sind dynamische egozentrierte Netzwerke, auch in Gestalt von Beziehungen zu unterschiedlichen Mentor/ innen bzw. Führungskräften, zu Peers wie anderen Mentees oder Kolleg/innen. Aktuell ist ein Verständnis von Mentoring als wechselseitige Lern- und Entwicklungsbeziehung, die sich in Richtung vielfältig vernetzter Lernbeziehungen im Umfeld öffnen. Im Zentrum des Interesses stehen sog. „high-quality relationships“, die frei von einer Reziprozitätsnorm wechselseitigen Nutzens ökonomischer oder symbolischer Art realisiert werden und eine hohe Bedeutung für Lernen, Führungskräfteentwicklung und Organisationslernen haben (Dutton und Heaphy 2003). Sie werden unter dem Begriff „relational mentoring“ diskutiert. In solchen (beidseitigen) Beziehungen sind Wirkungen des Mentorings auch für Mentor/innen zu erwarten, während in Bezug auf traditionelle Mentoring-Verständnisse lediglich der Nutzen für Mentees thematisiert wurde. Derartige von Wertschätzung und Vertrauen getragene Beziehungen erfordern hohe Kompetenzen von beiden Gesprächspartner/innen, Mentor/ in und Mentee: Empathie, Bereitschaft, die Rollen situativ zu wechseln (vgl. ,reverse mentoring“, Murphy 2012), Feedback-Kompetenz, Zuhören-Können u. a.

Mit Blick auf Führungskräfte gilt Mentoring auch als in (transformationale) Führung integriertes Geschehen, darin ist dieses Beratungsformat dem Coaching in der Rolle der Führungskraft als Coach vergleichbar. In Gestalt von Mentoring-Programmen - in Deutschland überwiegend zur Frauenförderung in Führungspositionen eingesetzt - entspricht es mit seinem formellen Rahmen und der zeitlichen 
Befristung mit Zielvereinbarung und Evaluation durch eine steuernde Instanz dem Coaching durch externe Coachs im Rahmen einer Personalentwicklungsmaßnahme. Letztlich ist das, was konkret unter Mentoring verstanden wird, sehr abhängig vom Kontext, in dem es stattfindet, und den dort geltenden Bedingungen.

Auch Coaching ist ein Sammelbegriff für verschiedenste Beratungsformen zur beruflichen und/oder persönlichen Unterstützung: Einzel-, Team- und Systemcoaching; Business-, Live- und Gesundheitscoaching u.v.m. Es zielt letztlich auf die Weiterentwicklung von individuellen und kollektiven Lern- und Leistungsprozessen in primär beruflichen Anliegen ab, und zwar entwicklungsfördernd und/ oder problemlösend sowie auch Orientierung gebend (vgl. DBVC 2012) - vorwiegend für Führungskräfte bzw. Menschen mit besonderer Verantwortung. Mit Bezug auf kollektive Lernprozesse öffnet es sich somit qua definitionem auch für Lernprozesse und -beziehungen außerhalb des Einzelcoachings, in der Praxis können Kombinationen mit Mentoring und Training entstehen. Wie Mentoring (nach neuerem Verständnis) gilt auch Coaching als dialogischer Prozess (vgl. Mührel 2009). Er basiert auf einer vertrauensvollen Beziehung, wobei einer seiner grundlegenden Merkmale die Förderung von Selbstreflexion und die Erweiterung von Wahrnehmung, Erleben und Verhalten darstellt (DBVC 2012). Dies ist wiederum vergleichbar mit Mentoring.

Vor dem Hintergrund der notwendigen Professionalisierungsbemühungen in der Coaching-Szene wird Coaching aber hier im Unterschied zu Mentoring als professionelle Beratung verstanden. Von Coachs wird daher zu Recht mehr als Berufserfahrung und fundierte Beratungskompetenz erwartet, die in der Regel durch eine formale Qualifikation dokumentiert wird, nämlich kontinuierliche Praxisreflexion (Supervision) und ein interdisziplinäres Theorieinventar u. a. aus Psychologie, Organisations- und Managementlehre (DBVC 2012) - Anforderungen, wie sie an Mentor/innen bisher so anspruchsvoll nicht gestellt werden. Mentoring und Coaching unterscheiden sich also vor allem hinsichtlich ihres Professionalisierungsgrades. Letztlich ist Coaching eine bezahlte Dienstleistung, während Mentoring ohne monetäre Gegenleistung angeboten wird, auch wenn es nicht ausschließlich von altruistischen Motiven getragen wird (vgl. Allen 2007).

Hinsichtlich der Einschätzung der beiden Beratungsformate Mentoring und Coaching herrschen auf Basis der jeweiligen Fachliteratur wechselseitig einige begriffliche Engführungen und Fehleinschätzungen vor, die an dieser Stelle nicht weiter ausgeführt werden können (hierzu Höher 2014), vor allem, wenn versucht wird, Unterschiede zu konstruieren.

\section{Zur nachhaltigen Wirksamkeit von Mentoring}

Einige Schlussfolgerungen aus einer aktuellen Untersuchung zum nachhaltigen, vernetzten Lernen durch Mentoring (Höher 2014) sollen nun dazu dienen, den Blick auf Coaching in Anlehnung an die Entwicklungen in der Mentoring-Forschung zu erweitern.

Der Untersuchungsgegenstand war ein zweijähriges, EU-ko-finanziertes regionales Cross-Mentoring-Programm mit insgesamt 21 Tandems, die jeweils ein Jahr zusammen arbeiteten. Es richtete sich an angestellte wie selbstständige weibliche Führungs- bzw. - Nachwuchskräfte im Kontext kleiner und mittelständischer Unternehmen in einer Wirtschaftsregion mit vergleichsweise ungünstigen Rahmenbedingungen für Frauen. Es verfolgte folgende Ziele: a) einen Beitrag zum Abbau der Unterrepräsentanz von Frauen in Fach- und Führungspositionen; b) Unterstützung beim Aufbau einer begonnenen Selbstständigkeit und c) das Schaffen eines Wissensnetzwerks von Frauen in der Region.

Um die nachhaltige Wirksamkeit des Mentorings im Sinne einer Langzeitperspektive einzuschätzen, wurde sechs Jahre später eine Nacherhebung bei den ehemaligen Teilnehmerinnen durchgeführt.

Einige zentrale Fragen waren:

- Wie haben Mentees und Mentorinnen sich innerhalb von sechs Jahren nach Projektende beruflich verändert und bewerten sie das als Erfolg?

- Inwieweit führen sie die Veränderungen selbst auf die Auswirkungen des damaligen Mentorings zurück?

- Welchen Stellenwert hat das Mentoring in der beruflichen Biografie der Frauen?

- Wie ist es den ehemaligen Mentees und Mentorinnen gelungen, die im Mentoring erzielten Ergebnisse und Lernerfolge umzusetzen bzw. langfristig aufrecht $\mathrm{zu}$ erhalten oder zu nutzen?

Die sich an vorgeschaltete Telefoninterviews mit 33 der ehemaligen Teilnehmerinnen anschließenden qualitativen (episodischen) Interviews thematisierten sechs Fälle vertieft, welche die Variationsbreite im Feld abbildeten. Alle Interviewten konstruierten in ihren biografischen Erzählungen einen Zusammenhang zwischen dem Mentoring und ihrer heutigen beruflichen Situation, die lt. telefonischer Befragung in der Mehrzahl der Fälle auch objektiven Erfolg im Sinne einer höheren Führungsposition, mehr Einkommen, Umsatz oder Kunden abbildeten. Davon abweichend konzipierten die Interviewten Erfolg jedoch mehrheitlich anhand subjektiver Kriterien wie persönliche Zufriedenheit, Kongruenz mit persönlichen Werten und Lebenszielen, gelungene Work Life Balance und Lernen/herausfordernde Aufgaben. Die Ergebnisse bestätigten das o. g. und aus der Mentoring-Forschung extrahierte aktuelle Verständnis von Mentoring als einer wechselseitigen Lernbeziehung, 
die sich für weitere Lernanlässe und -beziehungen öffnet, und zwar sowohl aus der Perspektive einiger Mentees wie Mentorinnen. Folgende Aussagen zu Mentoring trafen auf Repräsentantinnen beider Rollen zu:

- Es ermöglichte Selbstreflexion, wobei der Erfahrung von Differenz dabei eine besondere Bedeutung zukommt.

- Es stärkte Selbstbewusstsein und Selbstwertgefühl: bei den Mentorinnen aufgrund des Erlebens von Selbstwirksamkeit, bei den Mentees durch psychosoziale Unterstützung.

- Es war eine Erfahrung (im Sinne von Dewey 1986) von (berufs-)biografischer Relevanz.

- Es war ein vernetztes Lernen, das auf zuvor erschlossene Lernkontexte Bezug nahm und innerhalb der Tandembeziehung wechselseitig sowie in Beziehung zu anderen Mentees bzw. Mentorinnen im Netzwerk und ebenfalls in Verschränkung mit anderen Weiterbildungsaktivitäten innerhalb wie außerhalb des Mentoring-Programmes stattfand.

- Es ermöglichte nachhaltiges Lernen, da hier erschlossene Kompetenzen in späteren Situationen aktiviert und angewendet wurden.

Speziell für die Mentorinnen erwies sich das Mentoring in ihrer eigenen Darstellung als Praxisfeld zur Erprobung von Führungs- und Coaching-Kompetenzen. Mentoring kann auch, wie deutlich wurde, eine kompensatorische Funktion hinsichtlich eines erlebten Mangels an weiblichen Vorbildern und guter Führung haben: Für die Mentees diente dazu das Rollenmodell der Mentorinnen, bei diesen fungierten andere Mentorinnen als Vorbild.

Die Ergebnisse dieser nicht repräsentativen Studie entsprechen im Wesentlichen denen der neueren MentoringForschung (vgl. Ragins und Kram 2007; Schell-Kiehl 2007). Hier werden im Zuge eines Wandels des Mentoringverständnisses und der Rollen sowie einer Erweiterung seiner Funktionen (psychosoziale, karrierebezogene, role modelling, Lernen, networking) zunehmend auch der Nutzen für Mentor/innen und nicht mehr nur für Mentees thematisiert. Alle diese Erfolge von Mentoring sind in vieler Hinsicht mit den evaluierten Ergebnissen für Coaching vergleichbar. Es stellt sich daher die Frage, inwieweit die Frage nach dem Nutzen für beide Beteiligte nicht auch im Zusammenhang mit Coaching relevant sein könnte.

\section{Perspektiven für Coaching?}

Eine die Entwicklung der Mentoring-Forschung nachzeichnende analoge Herangehensweise für die CoachingForschung wäre, die Effekte von Coaching nicht nur für Coachees, sondern auch für Coachs $\mathrm{zu}$ erforschen und damit auch Coaching weiter in Richtung des wechselsei- tigen Lernens zu denken. Dies wäre, zumindest in Bezug auf die Rolle der Führungskraft als Coach, eine interessante Perspektive für deren weitere berufliche Entwicklung und Lernbiografie sowie bezüglich Coaching im Sinne eines Beitrags zum organisationalen Lernen. Die Frage ist dann, was Coachs im und durch Coaching (implizit) lernen. Diese Frage wurde bisher in der Forschung nicht gestellt, in der Praxis ist sie aber ein Thema für die Supervision von (externen) Coachs. Aufgrund der vielen Parallelen zwischen Mentoring und Coaching (siehe oben) möchte ich daher anregen, hinsichtlich der Wirkfaktoren von Coaching nicht nur - wie bisher - die Voraussetzungen der Coachs und ihre Interventionen (sowie die Voraussetzungen des Coachees) für erfolgreiches Coaching zu thematisieren, sondern verschiedene, auch implizite Formen der Wissens- und Erfahrungsgenerierung „on the job“ des Coachings in Verschränkung mit den Lernprozessen des Coachees als gemeinsame oder parallele Lernprozesse in den Blick zu nehmen. Damit könnte als ganzheitlicher und vernetzter Ansatz ein vollständigeres Bild der Wirkfaktoren und Wirksamkeit von Coaching entstehen, ähnlich, wie er für Mentoring seit jüngerer Zeit entdeckt wird. Letztlich ergeben sich aus einem Ansatz des vernetzten Lernens noch weitere Anregungen, wie etwa die Verknüpfung von Coaching mit anderen Lernanlässen und Kontexten weiter zu untersuchen, auch als Transferforschung und in (berufs-)biografischer Perspektive. $\mathrm{Zu}$ bedenken ist dabei allerdings, dass sich diese Untersuchungsperspektive mit Blick auf die Mentor/innen erst aufgrund eines Wandels der Mentoring-Verständnisse in Richtung wechselseitiger Lernbeziehungen erweitert hat, während dieser Aspekt bisher in der „black box Coaching“ noch völlig unentdeckt ist.

Open Access Dieser Artikel wird unter der Creative Commons Namensnennung 4.0 International Lizenz (http://creativecommons. org/licenses/by/4.0/deed.de) veröffentlicht, welche die uneingeschränkte Nutzung, Verbreitung und Wiedergabe für beliebige Zwecke erlaubt, sofern Sie den/die ursprünglichen Autor(en) und die Quelle ordnungsgemäß nennen, einen Link zur Creative Commons Lizenz beifügen und angeben, ob Änderungen vorgenommen wurden

\section{Literatur}

Allen, T. (2007). Mentoring relationships from the perspective of the mentor. In B. R. Ragins \& K. E. Kram (Hrsg.), The Handbook of Mentoring at Work (S. 123-148). Thousand Oaks: Sage.

Bresser, F. (2012). Tendenzen in der Coaching-Praxis und CoachingForschung weltweit. In R. Wegener, A. Fritze \& M. Loebbert (Hrsg.), Coaching entwickeln. Forschung und Praxis im Dialog (S. 189-199). Wiesbaden: Springer VS.

Brockband, A., \& McGill, I. (2006). Facilitating reflective learning through mentoring \& coaching. London: Kogan Page.

Connor, M., \& Pokora, J. (2007). Coaching and mentoring at work. Developing effective practice. Maidenhead: McGraw Hill. International. 
DBVC (Deutscher Bundesverband Coaching). (2012). Coaching als Profession (4., erw. Aufl.). Osnabrück: DBVC.

Dewey, J. (1986). Erziehung durch und für Erfahrung. Stuttgart: Klett-Cotta.

Dutton, J. E., \& Heaphy, E. D. (2003). The power of high quality connections. In K. S. Cameron, J. E. Dutton \& R. E. Quinn (Hrsg.), Positive Organizational Scolarship (S. 261-278). San Francisco: Berrett-Koehler.

Garvey, R., Stokes, P., \& Megginson, D. (2009). Coaching and mentoring. Theory and practice. London: Sage.

Giacovelli, S., \& Goldkamp, S. (2009). Coaching - if it matters, measure it? Formen und latente Funktionen der Nutzenquantifizierung im Coaching. In K. Galdynski \& A. Kühl (Hrsg.), Black-Box Beratung? Empirische Studien zu Coaching und Supervision (S. 222256). Wiesbaden: VS Verlag für Sozialwissenschaften.

Greif, S. (2008). Coaching und ergebnisorientierte Selbstreflexion. Göttingen: Hogrefe.

Greif, S. (2009). Grundlagentheorien und praktische Beobachtungen zum Coachingprozess. In B. Birgmeier (Hrsg.), Coachingwissen. Denn sie wissen nicht, was sie tun? (S. 129-144). Wiesbaden: Springer VS.

Greif, S. (2012). Die wichtigsten Erkenntnisse aus der Coaching-Forschung für die Praxis aufbereitet. In R. Wegener, A. Fritze \& M.
Loebbert (Hrsg.), Coaching entwickeln. Forschung und Praxis im Dialog (S. 35-44). Wiesbaden: Springer VS.

Higgins, M., \& Kram, K. E. (2001). Reconceptualizing mentoring at work. A developmental network perspective. Academy of Management Review, 26(2), 264-288.

Höher, F. (2014). Vernetztes Lernen im Mentoring. Wiesbaden: Springer VS.

Künzli, H. (2006). Wirksamkeitsforschung im Führungskräftecoaching. In E. Lippmann (Hrsg.), Coaching. Angewandte Psychologie für die Beratungspraxis (S. 279-323). Heidelberg: Springer.

Künzli, H. (2009). Wirksamkeitsforschung im Führungskräfte-Coaching. Organisationsberatung, Supervision, Coaching, 16, 4-14.

Mührel, E. (2009). Coaching als Dialog. Eine anthropologische Grundlegung des Coachings. In B. Birgmeier (Hrsg.), Coachingwissen. Denn sie wissen nicht, was sie tun? (S. 73-92). Wiesbaden: Springer VS.

Murphy, W. (2012): Reverse mentoring at work. Human Resource Management, 51(4), 549-547. doi:10.1002/hrm.21489.

Ragins, B. R., \& Kram, K. E. (2007). The handbook of mentoring at work. Theory, research and practice. Thousand Oaks: Sage.

Schell-Kiehl, I. (2007). Mentoring. Lernen aus Erfahrung? Bielefeld: Bertelsmann. 